\title{
Linx
}

Revue des linguistes de l'université Paris X Nanterre

5 | 1994

La négation

\section{La négation en arabe}

\section{Moktar Djebli}

\section{(2) OpenEdition \\ Journals}

Édition électronique

URL : http://journals.openedition.org/linx/1227

DOI : 10.4000/linx.1227

ISSN : 2118-9692

\section{Éditeur}

Presses universitaires de Paris Nanterre

\section{Édition imprimée}

Date de publication : 1 juin 1994

Pagination : 323-329

ISSN : 0246-8743

\section{Référence électronique}

Moktar Djebli, «La négation en arabe », Linx [En ligne], 5| 1994, mis en ligne le 18 juillet 2012, consulté le 19 avril 2019. URL : http://journals.openedition.org/linx/1227 ; DOI : 10.4000/linx.1227

Ce document a été généré automatiquement le 19 avril 2019

Département de Sciences du langage, Université Paris Ouest 


\section{La négation en arabe}

\section{Moktar Djebli}

1 Nous traiterons, dans cet exposé, d'une manière succincte les différentes formes de la négation en arabe, notamment du point de vue syntaxique ; ne serait-ce que pour mettre en évidence certains aspects contrastifs avec le français ; sachant que les langues - aussi éloignées les unes des autres, et malgré les apparences - renferment bien des similitudes.

\section{Aspect du verbe arabe}

2 En arabe, le système verbal se présente essentiellement sous trois aspects :

3 I. L'accompli : qui exprime une action achevée. Il correspond au passé français, avec ses différentes formes (passé composé, passé simple, imparfait, etc...) :

(1) katab ${ }^{a} \operatorname{Karîm}^{u n}$... [Karim a écrit, écrivit, écrivait, avait écrit, etc... ;

II. L'inaccompli ${ }^{1}$ : qui indique une action en cours de réalisation (présent, futur ou conditionnel) :

(2) yaktubu $\operatorname{Karîm}^{u n}$... Karim écrit, écrira ou écrirait...] ;

III. \& l'impératif : qui exprime, comme en français, un ordre affirmatif ou négatif.

(3) utkub! [Ecris!].

\section{Aspect de la phrase arabe}

Quant à la phrase arabe, elle se présente sous deux formes :

5 I. La phrase verbale : qui, comme son nom l'indique, contient un verbe explicitement, un sujet et différents compléments.

(4) kataba Karîm $^{u n}$ risâlat $^{a n}{ }^{\text {ilâ sadîq }}{ }^{i n} \ldots$ [Karim a écrit une lettre à un ami....

6 On note la déclinaison finale du sujet [un, Karîm ${ }^{\text {un] }}$ (cas nominatif), du complément d'objet direct [an, risâlat $^{a n}$ ] (cas accusatif) et du complément d'objet indirect [in, sadîq ${ }^{\text {in }}$ ] (cas génitif).

7 Aussi une véritable phrase arabe se présente-t-elle généralement de la sorte, c-à-d : 
verbe + sujet + complément d'objet direct + complément d'objet indirect, etc...

II. La seconde phrase est la phrase nominale : qui - elle - se compose généralement de deux éléments : un nom, ayant pour fonction grammaticale sujet et un attribut.

(5) Karîm ${ }^{u n} k_{a ̂ t i b}{ }^{u n}$. [Karim est un écrivain].

9 On relève que le verbe être est inexprimé, dans pareil cas. La flexion du sujet, tout comme celle de l'attribut, est [un] (cas nominatif).

\section{La négation}

10 La négation s'effectue différemment suivant le type de phrase et le temps exprimé.

\section{La phrase verbale :}

\section{i. La phrase verbale simple :}

11 Dans une phrase verbale simple, on fait appel à de nombreux morphèmes dépendamment du temps employé. Les plus usités sont les suivants :

A) Pour l'accompli (le passé) :

1. La particule la plus fréquemment utilisée est [mâa] (ne...pas).

(6) a. katab $^{a}$ Karîm $^{\text {un }}$ risâlat $^{a n}$. [Karim a écrit une lettre].

b. $\boldsymbol{m a} \boldsymbol{k a t a b}^{a} \boldsymbol{K a r i ̂ m}^{u n}{ }^{\text {risâlat }}{ }^{a n}$. [Karim n'a pas écrit une lettre] ${ }^{2}$

12 Il est à noter que la particule de la négation [mâ] précède le verbe, qui lui-même devance le sujet, comme nous l'avons déjà souligné.

13 2. Accompagnée d'autres morphèmes tels que [illâ, ghayr ou siwâa], cette particule [mâa] rend la négation française restrictive : $\boldsymbol{n} \boldsymbol{e} . . . q u e$, avec un temps passé.

(7) a. $\boldsymbol{m} \hat{a}$ katab $^{a}$ Karîm $^{u n}$ illâ risâlat ${ }^{a n}$. [Karim n'a écrit qu'une lettre.]

b. mâ katab ${ }^{\text {a }}$ Kầm ${ }^{\text {un }}$ siwâ (ou gayr ${ }^{\text {a }}$ ) risâlat ${ }^{\text {in }}$.

14 On note la déclinaison différente du complément, entrainée par l'intervention de ses deux particules (risâlatin au lieu de risâlatan).

3. Une seconde particule [lâ] (non, ne...pas) pourrait être employée avec un accompli, mais lorsqu'il s'agit précisément d'un vœu ou d'une malédiction.

(8) a. dâm ${ }^{a} l$-qitâl ${ }^{u} \ldots$ [Le combat a duré...]

b.lâ dâm ${ }^{a}$ l-qitâl ${ }^{u}$ [Que le combat ne dure pas!]

(9) a. rahim ${ }^{a} h^{u} L l a ̂ h^{u}$ ! [Que Dieu lui accorde sa bénédiction!].

b. lâ rahim ${ }^{a}-h^{u} L l a ̂ h^{u !}$ [Que Dieu ne lui accorde point sa bénédiction!].

B) Avec un verbe à l'inaccompli (le présent ou le futur) :

1. C'est même particule [lâa], qui est le plus souvent usitée.

(10) a. yaktub ${ }^{u}$ Karîm $^{u n}{ }^{u i s a ̂ l a t} t^{a n}$. [Karim écrit (ou écrira) une lettre.].

b. lâ yaktub ${ }^{u}$ Karîm $^{u n}$ risâlatan. [Karim n'écrit (ou n'écrira) pas une lettre.].

15 2. L'adverbe ne...que, peut être rendu également par l'association de cette particule [lâ] à une autre du groupe de [illâ] à l'instar de [mâa], comme nous l'avons précisé plus haut, mais avec un verbe à l'inaccompli.

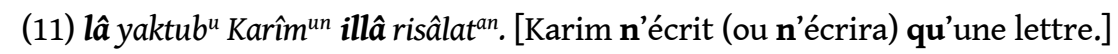


3. Encore le même morphème [lâ $]$ pourrait être employé pour exprimer une prohibition (un impératif négatif).

(12) a. takdhibu! [Tu mens.].

b. lâ takdhib! [Ne mens pas!].

[lâa] exige, dans ce cas, la disparition de la voyelle finale $[\mathbf{u}]$ du verbe takdhib ${ }^{u}$, transformé en : takdhib; ce que les arabisants désignent par le terme [sukûn] ou absence de voyelle, marquant l'apocopé (cf. note 2, p. 3).

17 4. Une dernière particule [lan] est utilisée pour exprimer, dans un temps futur, une négation catégorique et irrévocable.

(13) a. lâ yaktub ${ }^{u}$ Karîm $^{u n}$ risâlat $^{a n}$. [Karim n'écrit, n'écrirait, ou n'écrira pas une lettre], sans affirmation, ni assurance.

b. lan yaktub ${ }^{a}$ Karîm $^{u n}$ risâlat $^{a n}$. [Karim n'écrira pas une lettre]. Il s'agit d'une certitude, cette fois-ci ; car Karim, pour une raison ou une autre, n'écrira assurément pas de lettre. On relève ici la flexion verbale finale [ $\boldsymbol{a}$ au lieu de $\boldsymbol{u}$ ], entraînée par l'intervention de la particule [lan].

\section{ii. La phrase verbale complexe :}

Dans une phrase verbale complexe, la négation est quasi identique à celle d'une prhase simple.

C'est encore les morphèmes: [mâa], [lâ] et [lan], qui sont les plus souvent employés, suivant le temps exprimé.

Par exemple, dans une subordonnée relative :

à l'accompli (le passé), on a recours à [mâ]

(14) a. wasal ${ }^{a}$-rajul ${ }^{u}$ lladhî 'Araft ${ }^{u}$-hu. [L'homme, que j'ai connu, est arrivé].

b. mâ wasal ${ }^{a}$-rajul ${ }^{u} \underline{l l a d h \hat{\imath}}$ 'Araft ${ }^{u}$-hu. [L'homme, que j'ai connu, n'est pas arrivé].

21 On remarque que la particule de négation [mâ] est en tête de la proposition principale, comme dans une phrase verbale simple; [lladhî] étant le pronom relatif, introduisant la subordonnée.

à l'inaccompli, c'est la particule [lâ], qui est la plus fréquemment usitée :

(15) a. yasil ${ }^{u} r$-rajul ${ }^{u}$ lladhî 'Araft ${ }^{u}$-hu. [L'homme, que j'ai connu, arrive].

b. lâ yasil ${ }^{u}$ r-rajul ${ }^{u}$ lladhî 'Araft ${ }^{u}$-hu. [L'homme, que j'ai connu, n'arrive pas].

- et au futur, c'est le morphème [lan], qui est employé généralement :

(16) lan yasil ${ }^{u}$ r-rajul l'lladhî 'Araft ${ }^{u}$-hu. [L'homme, que j'ai connu, n'arrivera pas].

La négation française $\boldsymbol{n i} . . . n \boldsymbol{n}$, dans une coordination, est rendue souvent en arabe par les mêmes [mâ, lâ ou lan] et [lâa] conjointement [wa] (et).

(17) a. mâ shariba Karîm $^{u n}$ qahwat $^{a n}$, wa lâ shây ${ }^{a n}$. [Karim n'a bu ni café ni thé] (passé).

b. lâ yasharab ${ }^{u}$ Karîm $^{u n}$ qahwat $^{a n}$, wa lâ shây ${ }^{a n}$. [Karim ne boit ni café ni thé] (présent).

c. lan yasharab ${ }^{a}$ Karîm $^{\text {un }}$ qahwat $^{\text {an }}$, wa lâ shây ${ }^{a n}$. [Karim ne boira, certainement, ni café ni thé] (futur négatif catégorique).

\section{La phrase nominale}

En ce qui concerne la phrase nominale, il existe différentes formes de négation, dont les plus fréquentes sont les suivantes :

A) Au présent, la négation s'effectue à l'aide de : 
1. La particule [mâ], ou du verbe [laysa] qui exprime une négation d'existence (ne pas être).

(18) a. Karîm ${ }^{u n}$ kâtib $^{u n}$. [Karim est un écrivain (ou secrétaire)].

b. $\boldsymbol{m} \hat{\boldsymbol{a}}$ (ou laysa) Karîm ${ }^{\text {un }}$ kâtib $^{\text {an }}$. [Karim n'est pas un écrivain (ou secrétaire)].

L'introduction de $\boldsymbol{m} \hat{\boldsymbol{a}}$ (ou laysa) entraîne la voyelle double [tanwîn] à la fin de l'attribut [ kâtiban].

2. La particule [lâa] est également utilisée, dans pareil cas, mais dans un moindre mesure, afin d'exprimer une négation d'espèce ou de genre :

(19) a. fi d-dâri rajul ${ }^{\text {un }}$. [il y a un homme (masculin) à la maison].

b. lâ rajul ${ }^{a}$ fì $d$-dâri [il n'y a pas d'homme à la maison ; autrement dit ; aucun homme du genre masculin n'est à la maison ; mais des femmes, probablement].

On note, dans cette structure, la voyelle finale du nom $\left[\right.$ rajul $\left.^{a}\right]$ imposée par l'intervention de $[\mathbf{l a}]$.

B) Au passé, la négation dans une phrase nominale, s'obtient, essentiellement, par le biais de la particule [mâa], accompagnée d'un verbe d'état [kâna] employé à l'accompli comme exposant temporel et rendu souvent, en français, par le verbe être à l'imparfait ou au plus-que-parfait.

(20) a.kân ${ }^{a}$ Karîm $^{u n}$ kâtib ${ }^{a n}$. [Karim était un écrivain (ou secrétaire)].

b. $\boldsymbol{m a ̂}$ kân $^{a}$ Karîm $^{\text {un }} k \hat{a} t i b^{a n}$. [Karim n'était pas un écrivain].

Il est à noter que, bien que la phrase contienne un verbe, elle est toujours considérée comme une phrase nominale, mais introduite par le verbe d'état [kâna]. Quant à la déclinaison, elle est identique à celle de [laysa] ([un] pour le sujet: Karîm ${ }^{u n}$ et [an] pour l'attribut : kâtibin ), comme nous l'avons signalé plus haut (cf. p.327).

C) Pour le futur :

25 1) On a souvent recours au même verbe [kâna], mais conjugué à l'inaccompli et accompagné de la particule [lậ], employée dans une négation verbale au présent.

(21) a. yakûn ${ }^{u}$ Karîm $^{u n}$ kâtib ${ }^{a n}$. [Karim sera (ou serait) un écrivain].

b. lâ yakûn ${ }^{u}{\text { Karîm }{ }^{u n}}^{u a ̂ t i b a n}$. [Karim ne sera (ou ne serait) pas un écrivain].

26 2) La même forme pourraît être employée, mais en substituant [lan] à [lâ], pour exprimer une négation future, plus catégorique.

(22) lan yakûn ${ }^{a}$ Karîm $^{u n}$ kâtiban. [Karim ne sera (certainement) pas un écrivain].

3. En revanche, cette même phrase, introduite par [lâa], pourrait être exprimée au conditionnel et marquant ainsi un doute, quant à la réalisation de l'action. Pour ce faire, il suffit de l'introduire par un adverbe de doute [gad].

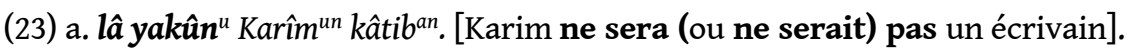

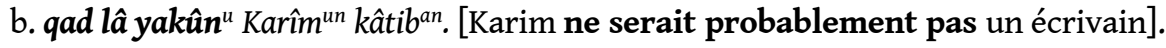

D'autres verbes d'état ou d'existence, dits du groupe de [kâna], une quinzaine en tout, ont le même usage que celui-ci.

(24) a. $\boldsymbol{m} \hat{\boldsymbol{a}} z \hat{\boldsymbol{a}} \mathbf{l}^{a} \mathrm{Karîm}^{u n}$ kâtib $^{a n}$. [Karim ne cesse d'être un écrivain] ; ou avec [lâ] :

b. lâ ya zâl ${ }^{a}$ Karîm $^{u n}$ kâtiban. [Karim ne cessera d'être un écrivain]. (V. à ce sujet, notamment: Afghânî, Mudhakkarât fî l-qawầid: pp. 20sq ; Blachère, Eléments de l'arabe classique : pp.44sq., Djebli, Eléments de grammaire arabe : p. 15).

Encore une dernière remarque à propos de ces particules (mâ, lâ et lan). En effet, comme pour la phrase verbale, elles peuvent être employées accompagnées de [illâ, ghayr ou 
siwâa], déjà citées (cf. p. 3), dans une phrase nominale exceptive, exprimée en français par la négation restrictive : $\boldsymbol{n e}$...que.

a) Au présent :

(25) $\boldsymbol{m} \hat{a}$ Karîm $^{u n}$ illâ kâtib ${ }^{a n}$. [Karim n'est qu'un écrivain],

b) Au passé, dans une phrase introduite par le verbe d'état [kâna] :

(26) $\boldsymbol{m} \hat{a}$ kân ${ }^{a} K$ rrîm $^{u n}$ illâ kâtiban . [Karim n'était qu'un écrivain],

c) A l'inaccompli :

- Futur ou conditionnel :

(27) lâ yakûn ${ }^{u} K$ rrîm $^{u n}$ illâ kâtiban. [Karim ne sera (ou ne serait) qu'un écrivain], - Ou, enfin, futur négatif avec certitude :

(28) lan yakûn ${ }^{a} K^{\prime} \hat{r}^{u n}{ }^{u n}$ illâ kâtib $^{a n}$. [Karim ne sera, certainement, qu'un écrivain].

\section{NOTES}

1. Certains emploient, à ce propos, les termes perfect et imperfect, termes inadéquats, à notre avis. Les arabisants, quant à eux, préfèrent en général les deux autres termes (accompli \& inaccompli).

2. Deux autres particules sont également employées d'une façon quasi identique à $[\boldsymbol{m} \hat{a}]$; il s'agit de [lam et lammâ $]$. Leur emploi est cependant plus complexe. Elles exigent, en effet, un mode de verbe, que les arabisants francophones dénomment apocopé.

a. kata Karîmun risâlatan. [Karîm a écrit une lettre].

b. lam yaktub Karimun risâlatan. Ceci équivaut à la phrase : mâ kataba Karîmun risâlatan. (Karîm n'a pas écrit une lettre).

Une nuance, toutefois, entre [lam] et [lammâ $]$; si la première exprime une négation au passé, la seconde [lammâ] exprime une négation constante, continue jusqu'au présent.

c. lammâ yaktub Karimun risâlatan. [Karîm n'a pas encore écrit une lettre].

\section{RÉSUMÉS}

La négation arabe s'effectue différemment suivant deux facteurs : le type dd phrase et le temps exprimé.

I - Dans une phrase verbale, on fait appel à de nombreuses particules ainsi :

A) Pour l'accompli [le passé] : la particule la plus fréquemment utilisée est [mâ] ou [lam] (ne... pas) (ex. 6).

B) Avec un verbe à l'inaccompli (le présent ou le futur) : deux autres particules sont souvent employées [lâ] et [lan], avec cependant une nuance ; car cette dernière exprime une négation catégorique certaine (ex. 10 \& 13).

II - En ce qui concerne la phrase nominale, différentes formes de négation sont usitées :

A) Au présent, la négation s'effectue à l'aide du morphème [mâ], ou du verbe [laysa] qui exprime 
une négation d'existence (ne pas être) (ex. 18).

B) Au passé, la négation s'obtient, essentiellement, par le biais de la particule [mâ], accompagnée d'un verbe d'état [kâna] employé à l'accompli comme exposant temporel et rendu souvent, en français, par le verbe être à l'imparfait ou au plus-que-parfait (ex. 20).

C) Pour le futur: on a souvent recours au même verbe [kâna], mais conjugué à l'inaccompli et accompagné de la particule [lâ] ou bien [lan], s'il s'agit d'une négation catégorique et irrévocable (ex. $21 \& 22$ ).

Negation in Arabic is used differently according to two factors: the type of the sentence and the tense.

I - In a verbal sentence:

A) With the past tense (perfect): two particles are frequently used: [mâ] and [lam] (not...) (ex. 6).

B) With the present or the future: two others adverbs are equally used: [lâ] and [lan]; the latter nevertheless indicates a definite negation specially in the future (ex. 10 \&13).

II - In a nominal sentence:

A) In the present tense: negation is expressed by means of the adverb [mâ], or the verb [laysa] which is a negation of existence (not to be) (ex. 18).

B) In the past tense: negation is essentially obtained by the means of the same particle [mâ], accompanied by the verb of state [kâna], in the preterit (ex. 20).

C) In the future tense: we use the same verb [kâna], but the present is accompanied by the particle [lâ], or [lan] if at concerns a categorical and irrevocable negation (ex. 21 \& 22).

\section{AUTEUR}

\section{MOKTAR DJEBLI}

Maître de Conférences, Université Paris X - Nanterre 